\section{Grippeimpfung bei Senioren: Welche Variablen beeinflussen die Wirksamkeit?}

Darvishian M et al. Effectiveness of seasonal influenza vaccination in community-dwelling elderly people: an individual participant data meta-analysis of test-negative design casecontrol studies. Lancet Respir Med 2017; 5: $200-211$

Weltweit empfehlen Impfkommissionen die Grippeschutzimpfung vor allem für Menschen ab 60 Jahren, Personen mit Diabetes mellitus oder Asthma, medizinisches Personal und Betreuer von Risikopatienten. Eine niederländische Metaanalyse untersuchte nun Test-negative Fall-Kontroll-Studien nach Störfaktoren wie Geschlecht, Alter und chronische Krankheiten, welche die Wirksamkeit der Grippeimpfung bei selbstständig lebenden Senioren beeinflussen könnten.

Für ihre Metaanalyse berücksichtigten die Autoren individuelle Teilnehmerdaten aus Test-negativen Fall-Kontroll-Studien früherer Metaanalysen, die bis einschließlich Mitte Juli 2014 publiziert wurden. Die Forscher hatten Zugang zu den individuellen Teilnehmerdaten. Patienten, die nicht eindeutig identifiziert werden konnten, deren Impfstatus sowie Ausgang unbekannt war oder jene mit mehr als einem Verdacht auf eine Influenzainfektion in derselben Saison, wurden aus der Analyse ausgeschlossen. Patienten mit grippeähnlichen Sympto- men, die für mindestens einen der folgenden Erreger positiv getestet waren, gingen in die Fallgruppe ein: $\mathrm{A}(\mathrm{H} 1 \mathrm{~N} 1)$, A (H1N1) pdm09, A(H3N2) oder B-Virus. Menschen mit influenzaähnlichen Symptomen und negativem Virustest bildeten die Kontrollgruppe. Als primärer und sekundärer Endpunkt galt die Wirksamkeit der Grippeimpfung einer labordiagnostisch bestätigten Grippe. Sie wurde als relative Risikoreduktion bei geimpften Personen definiert, verglichen zu nicht geimpften Patienten. Die Subgruppenanalyse zur Impfstoffwirksamkeit berücksichtigte außerdem Hemisphäre, Alterskategorie und Gesundheitsstatus.

\section{Ergebnisse}

In die Metaanalyse gingen 23 der 53 erhaltenen Datensätze ein. Weitere $6 \mathrm{Da}$ tensätze stellten Studienkollaborateure bereit. Insgesamt waren es 5210 Studienteilnehmer. Die Einschlusskriterien erfüllten 4975 Patienten, davon waren $63 \%$ in der Kontroll- und $37 \%$ in der Fallgruppe. Die saisonale Grippeimpfung war, unabhängig vom gematchten Impfstatus, signifikant gegen die epidemische Grippe wirksam. Während der nicht epidemischen Saison war diese Signifikanz nicht gegeben. Die Wirksamkeit der Grippeimpfung war stark vom Virustyp und -subtyp abhängig. Die höchste geschätzte Wirksamkeit der Impfung gab es mit $53,19 \%$ gegen $A(\mathrm{H} 1 \mathrm{~N} 1)$ pdm09-Viren, und die niedrigste gegen den Influenza B-Virus (-1,52\%). Die Subgruppenanalyse ergab keine signifikanten Unterschiede. Allerdings zeigte die Grippeimpfung einen protektiven Effekt für selbstständig lebende Senioren mit kardiovaskulären Erkrankungen, Lungenerkrankungen und bei Senioren bis 75 Jahren.

FAZIT

Laut Meinung der Autoren hatte die Grippeimpfung bei älteren Personen in dieser Metaanalyse einen moderaten Effekt. Dieser war während der epidemischen Saison und vor dem Hintergrund einer labordiagnostisch bestätigten Influenza gegeben. Weitere Untersuchungen seien nötig, um beeinflussende Faktoren für den Impfschutz zu identifizieren. Dazu könnten zum Beispiel markenspezifische oder typenspezifische Impfstoffe gehören sowie der Effekt einer jährlichen Grippeimpfung bei älteren Menschen.

Dr. Marion Rukavina, Berlin 